\title{
Detection of Coherent Signals Using Weighted Subspace Smoothing
}

\author{
Ching-Wen Ma, Student Member, IEEE, and Ching-Cheng Teng
}

\begin{abstract}
A new approach to detecting the number of coherent signals incident upon a uniformly-spaced linear array is presented. The approach combines a modified spatial smoothing scheme and a modified MDL criterion. The modified spatial smoothing scheme, referred to as "weighted subspace smoothing," is actually a generalization of the "post-smoothing" approach proposed by Krim and Proakis in 1994. It is shown that the noise eigenvalues obtained with weighted subspace smoothing are more accurate than those obtained with the original spatial smoothing. We thus attempt to improve detection performance using these more accurate eigenvalues. A novel modification of the minimum description length (MDL) criterion is proposed to accomplish this. Computer simulations are presented to study the performance improvement of this new approach.
\end{abstract}

\section{INTRODUCTION}

A RRAY signal processing deals with extracting information from measurements collected by an array of sensors. An important issue in this field is detecting how many signals impinge on the array. Once the number of signals is detected, many high-resolution direction-of-arrival (DOA) estimation methods can be used [1]-[4].

One way to solve the detection problem is based on hypothesis testing using eigenvalues of observed-vector covariance matrix [5], [6]. A disadvantage of this method is the subjective judgment required in choosing the threshold. To avoid this, Wax and Kailath [9] proposed an approach based on Akaike information criterion (AIC) [7] and SchwartzRissanen's minimum description length (MDL) criterion [8]. Assuming spatially-white Gaussian noise is additive, they obtained two different criteria. The number of signals is determined as the value for which the AIC or the MDL criterion is minimized. Further investigation [10]-[15] showed that the MDL criterion is consistent, while the AIC criterion exhibit better performance at a relatively lower SNR or with a smaller number of snapshots, at the cost of being inconsistent. Since consistency is important in detection, the MDL criterion seems to be more popular. There is an annoying problem with both criteria, however; the detection performance suffers serious degradation when some signals are coherent or highly correlated. To overcome this, Wax and Ziskind [19], Wax [20], Wu and Fuhrmann [21], Cho and Djurić [22], and Viberg et al. [29] presented methods that determined the number of signals based on signal DOA estimates. Since

Manuscript received November 14, 1994; revised May 17, 1995. This work was supported by the National Science Council, R.O.C. under Grant NSC 85-2212-E-009-028.

The authors are with the Department of Control Engineering, National Chiao-Tung University, Hsinchu, Taiwan, R.O.C..

Publisher Item Identifier S 0018-926X(96)01199-4. coherent signal DOA estimates are normally obtained by multidimensional minimization, these methods generate high computational loading. For a uniformly-spaced linear array (ULA), multidimensional minimization can be avoided by using the iterative quadratic maximum likelihood approach (IQML) [26] or the method of direction estimation (MODE) [27]. However, IQML and MODE both require that the number of signals be known in advance. Another solution is the spatial smoothing approach, which "decorrelates" coherent signals. In [18], Shan et al. applied the MDL criterion to a spatiallysmoothed covariance matrix to alleviate the computational loading.

In this paper, we present a new approach that exhibits better detection performance than directly applying the MDL criterion to the spatially-smoothed covariance matrix and does not require DOA estimation. The approach combines a modified spatial smoothing scheme and a modified MDL criterion. The modified spatial smoothing scheme applies the original spatial smoothing algorithm to an estimate of the noise-free covariance matrix. Since we estimate the noisefree covariance matrix by using the weighted signal subspace, we call the resulting modified spatial smoothing scheme "weighted subspace smoothing" (WSS). WSS is actually a generalization of the "post-smoothing" approach proposed by Krim and Proakis [30]. By "post-smoothing," they mean the smoothing transformation is applied to a well-defined reduced-rank approximation of the array covariance matrix. The reduced-rank approximation can be obtained using WSS with a particular weighting matrix. In this paper, we estimate the noise-free array covariance matrix using WSS with another weighting matrix. After the noise-free estimate is obtained, WSS performs the original spatial smoothing algorithm on it and obtains an estimate of the noise-free spatially-smoothed covariance matrix, which is denoted by $\hat{\tilde{\mathbf{R}}}_{x}$. Here, " " " denotes estimated and " $\sim$ " denotes application of spatial smoothing algorithm to the noise-free covariance matrix. The noise eigenvalues of $\hat{\tilde{\mathbf{R}}}_{x}$ have been shown to be more accurate than those obtained with the original spatial smoothing scheme. We thus attempt to improve detection performance by using these more accurate eigenvalues. A novel modification of the MDL criterion is used to accomplish such purpose. Though the modification is simple, simulation results demonstrate that detection performance is improved considerably.

Note that in [29], Viberg et al. proposed a method named weighted subspace fitting (WSF) detection scheme for signal detection. It is based on the asymptotic distribution of a 
well-defined WSF cost function, which is constructed using the weighted signal subspaces. Because the scheme uses the weighted signal subpaces to improve the coherent signal detection for the first time, we briefly review the scheme. First, the scheme makes an assumption on the number of signals. Then, it uses ESPRIT [3] or the alternating projection method [4] to obtain initial estimates of DOA's and uses a Gauss-Newton type algorithm to minimize the WSF cost function. Finally, it decides whether to accept this assumption or not by comparing the minimized WSF cost function with a judiciously selected threshold. Actually, the WSF detection scheme combines the problems of signal-detection and DOAestimation as a whole. Although the WSF detection scheme is much more complicated than the proposed method described in previous paragraph, computer simulations show that its performance is inferior, especially when more signals are coherent.

The paper is organized as follows. First, the problem is formulated and the method combining spatial smoothing and MDL (or AIC) criterion is reviewed in Section II. In this section, we also present the concept of WSS. In Section III, the accuracy of the eigenvalues of $\hat{\tilde{\mathbf{R}}}_{x}$ is discussed and modified MDL criterion is then introduced to take advantage of this accuracy. In Section IV, detection performance is examined by simulations. In the last section, we present our conclusions.

\section{PROBLEM FORMULATION}

In this section, we first present the signal model and summarize the MDL criterion. We then point out the difficulty of applying the MDL criterion when some signals are coherent and outline how spatial smoothing can overcome the difficulty. Finally, we focus on weighted subspace smoothing, which we believe to be more efficient for both DOA-estimation and signal-detection problems.

\section{A. Signal Model and MDL Criterion}

Considering narrow-band signals, the output of an array with $m$ sensors is commonly modeled as follows

$$
\mathbf{x}(t)=\mathbf{A}(\Theta) \mathbf{s}(t)+\mathbf{n}(t)
$$

where $\mathbf{x}(t)$ is an $m \times 1$ complex observation vector, $\mathbf{s}(t)$ is a $p \times 1$ vector that denotes the complex envelopes of $p$ signals, $\mathbf{A}(\Theta)$ is an $m \times p$ matrix whose columns are the direction vectors with parameters $\Theta$ denoting the angles of arrival of the $p$ signals, and $\mathbf{n}(t)$ is an $m \times 1$ vector representing receiver noise.

It is assumed that $\mathbf{s}(t)$ and $\mathbf{n}(t)$ are stationary and ergodic, complex-valued normal random processes having zero means, $\mathbf{n}(t)$ is uncorrelated with $\mathbf{s}(t)$, and the covariance matrix of $\mathbf{n}(t)$ is $\sigma^{2} \mathbf{I}$. Here, $\sigma^{2}$ is noise power at each sensor, and $\mathbf{I}$ is an identity matrix. Based on these assumptions, the covariance matrix of $\mathbf{x}(t)$ has the form

$$
\begin{aligned}
\mathbf{R}_{x} & \triangleq E_{i}\left[\mathbf{x}(t) \mathbf{x}(t)^{H}\right] \\
& =\mathbf{A}(\Theta) E\left[\mathbf{s}(t) \mathbf{s}(t)^{H}\right] \mathbf{A}(\Theta)^{H}+\sigma^{2} \mathbf{I} \\
& =\mathbf{A}(\Theta) \mathbf{R}_{s} \mathbf{A}(\Theta)^{H}+\sigma^{2} \mathbf{I}
\end{aligned}
$$

where $E[y]$ is the mean of $y,(\cdot)^{H}$ denotes conjugate transpose, and $\mathbf{R}_{s} \triangleq E\left[\mathbf{s}(t) \mathbf{s}(t)^{H}\right]$. Computing the eigen-decomposition of $\mathbf{R}_{x}$, we obtain

$$
\mathbf{R}_{x}=\sum_{k=1}^{m} \lambda_{k} e_{k} e_{k}^{H}
$$

where $\lambda_{1} \geq \lambda_{2} \geq \cdots \geq \lambda_{m}$. Let $p^{\prime}$ denotes the rank of $\mathbf{R}_{s}$. We have $p^{\prime} \leq p$. It follows from simple rank-property implications [1] that the last $m-p^{\prime}$ eigenvalues are all equal to $\sigma^{2}$, i.e.,

$$
\lambda_{p^{\prime}+1}=\lambda_{p^{\prime}+2}=\cdots=\lambda_{m}=\sigma^{2} .
$$

If $p^{\prime}=p$, we can determine the number of signals by computing how many eigenvalues are greater than the smallest eigenvalue.

In practical applications, the exact ensemble covariance matrix $\mathbf{R}_{x}$ is not known. Nonetheless, we are given a series of samples from $\mathbf{x}(t)$, say $\left\{\mathbf{x}\left(t_{1}\right), \mathbf{x}\left(t_{2}\right), \cdots, \mathbf{x}\left(t_{N}\right)\right\}$. Our goal, here, is to estimate $p$ using these samples. A solution to this problem lies in using an estimate of the covariance matrix instead of the exact one. The maximum-likelihood estimate of $\mathbf{R}_{x}$, the sample covariance matrix $\hat{\mathbf{R}}_{x}$, is computed as follows:

$$
\hat{\mathbf{R}}_{x}=\frac{1}{N} \sum_{i=1}^{N} \mathbf{x}\left(t_{i}\right) \mathbf{x}\left(t_{i}\right)^{H}
$$

The eigen-decomposition of $\hat{\mathbf{R}}_{x}$ is

$$
\hat{\mathbf{R}}_{x}=\sum_{k=1}^{m} \hat{\lambda}_{k} \hat{e}_{k} \hat{e}_{k}^{H}
$$

Now, $\hat{\lambda}_{1}>\hat{\lambda}_{2}>\cdots>\hat{\lambda}_{m}$ with probability 1 for finite $N$. The detection of $p^{\prime}$ becomes an interesting problem. In [9], Wax and Kailath treated it as a model selection problem and applied the information theoretic criteria developed by Akaike (AIC) and by Rissanen (MDL) to it. After substituting maximum-likelihood estimates of the eigenvalues to a loglikelihood function, they concluded that the AIC and the MDL criteria could be simplified as

$$
\operatorname{AIC}(k)=N(m-k) \log \left(\frac{a(k)}{g(k)}\right)+k(2 m-k)
$$

and

$$
\operatorname{MDL}(k)=N(m-k) \log \left(\frac{a(k)}{g(k)}\right)+\frac{1}{2} k(2 m-k) \log N
$$

respectively, where

$$
\begin{aligned}
& a(k) \triangleq \frac{1}{m-k} \cdot \sum_{i=k+1}^{m} \hat{\lambda}_{i} \\
& g(k) \triangleq\left(\prod_{i=k+1}^{m} \hat{\lambda}_{i}\right)^{1 /(m-k)}
\end{aligned}
$$

The estimate of $p^{\prime}$, denoted by $\hat{p}^{\prime}$, is determined by the value of $k \in\{0,1, \cdots, m-1\}$ which minimizes the AIC or the MDL criterion. In this paper, we concentrate on the MDL criterion, because it is consistent. 


\section{B. A Combination of Spatial Smoothing and MDL Criterion}

It is well known that $p^{\prime}<p$, when some signals are fully correlated or coherent. As a result, the methods presented above fail to detect fully correlated or coherent signals. Spatial smoothing enables the MDL criterion to deal with the problem, provided that the array is uniformly-spaced and linear. The array is grouped into several subarrays, which have the same geometry. If we employ the forward/backward spatial smoothing scheme, the estimated spatially-smoothed covariance matrix is given as follows

$$
\hat{\overline{\mathbf{R}}}_{x}=\frac{1}{2 K} \sum_{k=1}^{K} \mathbf{Z}_{k} \hat{\mathbf{R}}_{x} \mathbf{Z}_{k}^{T}+\mathbf{Q}_{k} \hat{\mathbf{R}}_{x}^{*} \mathbf{Q}_{k}^{T}
$$

where $(\cdot)^{T}$ denotes transpose, $(\cdot)^{*}$ denotes conjugate, and

$$
\begin{aligned}
\mathbf{Z}_{k} & =\left[\mathbf{0}_{(k-1) \times m^{\prime}}\left|\mathbf{I}_{m^{\prime} \times m^{\prime}}\right| \mathbf{0}_{(K-k) \times m^{\prime}}\right] \\
\mathbf{Q}_{k} & =\left[\mathbf{0}_{(k-1) \times m^{\prime}}\left|\mathbf{Q}_{m^{\prime} \times m^{\prime}}\right| \mathbf{0}_{(K-k) \times m^{\prime}}\right]
\end{aligned}
$$

the subscript indicates the dimension of a matrix, $\mathbf{0}$ is a zero matrix, and

$$
\mathbf{Q} \triangleq\left[\begin{array}{ccccc}
0 & \cdots & \cdots & 0 & 1 \\
0 & \cdots & 0 & 1 & 0 \\
\vdots & \vdots & & & \vdots \\
0 & 1 & 0 & \cdots & 0 \\
1 & 0 & \cdots & \cdots & 0
\end{array}\right]
$$

In the above equation, $m^{\prime}=m-K+1$ is the number of sensors of each subarray. It has been shown, in [24] and [25], that the exact ensemble spatially smoothed covariance matrix $\overline{\mathbf{R}}_{x}$ can be expressed as

$$
\overline{\mathbf{R}}_{x}=\overline{\mathbf{A}}(\Theta) \overline{\mathbf{R}}_{s} \overline{\mathbf{A}}(\Theta)^{H}+\sigma^{2} \mathbf{I}
$$

where $\overline{\mathbf{A}}(\Theta)$ is an $m \times p^{\prime}$ matrix consisting direction vectors. The rank of $\overline{\mathbf{R}}_{s}$, denoted by $\bar{p}^{\prime}$, is now equal to the number of signals, $p$, regardless of the signal coherency. Computing eigen-decomposition of $\hat{\mathbf{R}}_{x}$, we obtain

$$
\hat{\overline{\mathbf{R}}}_{x}=\sum_{k=1}^{m^{\prime}} \hat{\bar{\lambda}}_{k} \hat{\bar{e}}_{k} \hat{\bar{e}}_{k}^{H}
$$

where $\hat{\bar{\lambda}}_{1}>\hat{\bar{\lambda}}_{2}>\cdots>\hat{\bar{\lambda}}_{m^{\prime}}$. The number of signals could then be determined as the value, $k$, that minimizes the following criterion

$$
\overline{\operatorname{MDL}}(k)=N\left(m^{\prime}-k\right) \log \left(\frac{\bar{a}(k)}{\bar{g}(k)}\right)+\frac{1}{2} k\left(2 m^{\prime}-k\right) \log N
$$

where

$$
\begin{aligned}
& \bar{a}(k) \triangleq \frac{1}{m^{\prime}-k} \sum_{i=k+1}^{m^{\prime}} \hat{\bar{\lambda}}_{i} \\
& \bar{g}(k) \triangleq\left(\prod_{i=k+1}^{m^{\prime}} \hat{\bar{\lambda}}_{i}\right)^{1 /\left(m^{\prime}-k\right)} .
\end{aligned}
$$

Here, $k \in\left\{0,1, \cdots, m^{\prime}-1\right\}$. We designate the estimated number as $\hat{\bar{p}}^{\prime}$. In [18], Shan et al. used this method to determine the source coherency structure, showing that the method is suitable for detecting coherent signals. In this paper, we make modifications on both spatial smoothing algorithm and MDL criterion to improve detection performance.

Remark: $\hat{\mathbf{R}}_{x}$ is indeed formed by $2 K \cdot N$ vectors. One may suggest that the $N$ in (19) should be replaced by $2 K \cdot N$. However, if we do so, the criterion tends to overestimate the number of signals.

\section{The Weighted Subspace Smoothing}

Weighted subspace smoothing (WSS) "decorrelates" signal coherency by applying the smoothing transformation to the weighted signal subspace. Before introducing the scheme, we take a look at the eigen-decomposition of the covariance matrix $\mathbf{R}_{x}$, and define some notation. Following (5), we obtain

$$
\begin{aligned}
\mathbf{R}_{x} & =\mathbf{A}(\Theta) \mathbf{R}_{s} \mathbf{A}(\Theta)^{H}+\sigma^{2} \mathbf{I} \\
& =\mathbf{E}_{s} \boldsymbol{\Lambda}_{s} \mathbf{E}_{s}^{H}+\mathbf{E}_{n} \boldsymbol{\Lambda}_{n} \mathbf{E}_{n}^{H} \\
& =\mathbf{E}_{s}\left(\boldsymbol{\Lambda}_{s}-\sigma^{2}\right) \mathbf{E}_{s}^{H}+\sigma^{2} \mathbf{I}
\end{aligned}
$$

where $\mathbf{E}_{s} \triangleq\left[e_{1}, e_{2}, \cdots, e_{p^{\prime}}\right], \mathbf{E}_{n} \triangleq\left[e_{p^{\prime}+1}, e_{p^{\prime}+2}, \cdots, e_{m}\right]$, $\boldsymbol{\Lambda}_{s} \triangleq \operatorname{diag}\left(\lambda_{1}, \lambda_{2}, \cdots, \lambda_{p^{\prime}}\right)$, and $\boldsymbol{\Lambda}_{n} \triangleq \operatorname{diag}\left(\lambda_{p^{\prime}+1}, \lambda_{p^{\prime}+2}\right.$, $\left.\cdots, \lambda_{m}\right)=\sigma^{2} \mathbf{I}$. It is well known [1], that the range space of $\mathbf{E}_{s}$ is a subset of the range space of $\mathbf{A}(\Theta)$, i.e.,

$$
\text { Range }\left\{\mathbf{E}_{s}\right\} \subseteq \operatorname{Range}\{\mathbf{A}(\Theta)\}
$$

with equality if and only if $p^{\prime}=p$. The eigenvectors $\left\{e_{1}, e_{2}, \cdots, e_{p^{\prime}}\right\}$ are usually called the signal eigenvectors, and the range space of $\mathbf{E}_{s}$ is called the signal subspace. Its orthogonal complement is usually referred to as the noise subspace, which is spanned by the columns of $\mathbf{E}_{n}$. The eigendecomposition of the sample covariance matrix $\hat{\mathbf{R}}_{x}$ is defined in a similar fashion as (22)

$$
\hat{\mathbf{R}}_{x}=\hat{\mathbf{E}}_{s} \hat{\mathbf{\Lambda}}_{s} \hat{\mathbf{E}}_{s}^{H}+\hat{\mathbf{E}}_{n} \hat{\mathbf{\Lambda}}_{n} \hat{\mathbf{E}}_{n}^{H}
$$

Here, $p^{\prime}$ must be estimated using the MDL criterion, or other method, to construct $\hat{\mathbf{E}}_{s}, \hat{\mathbf{E}}_{n}, \hat{\mathbf{\Lambda}}_{s}$, and $\hat{\boldsymbol{\Lambda}}_{n}$. The signal component and the noise component are now separated. From (23), we know that the signal eigenvectors are linear combinations of direction vectors. Thus, we can decorrelate the coherent signals based on $\mathbf{E}_{s}$. One method of achieving this is to apply spatial smoothing to the following matrix

$$
\hat{\dot{\mathbf{R}}}_{x} \triangleq \hat{\mathbf{E}}_{s} \mathbf{W} \hat{\mathbf{E}}_{s}^{H}
$$

where $\mathbf{W}$ is a weighting matrix. If $\mathbf{W}$ is set to be $\hat{\boldsymbol{\Lambda}}_{s}$, then $\hat{\mathbf{R}}_{x}$ is a reduced-rank approximation of the array covariance matrix. In [30], the reduced-rank approximation is used to improve DOA-estimation accuracy. If $\mathbf{W}$ is set to be $\hat{\boldsymbol{\Lambda}}_{s}-\hat{\sigma}^{2} \mathbf{I}$, where

$$
\hat{\sigma}^{2} \triangleq \frac{1}{m-\hat{p}^{\prime}} \sum_{k=\hat{p}^{\prime}+1}^{m} \hat{\lambda}_{k}
$$

then $\hat{\dot{\mathbf{R}}}_{x}$ is an estimate of the noise-free array covariance matrix. We will improve detection performance using this 
$\hat{\dot{R}}_{x}$. Below, we summarize the weighted subspace smoothing scheme:

a) Compute the sample covariance matrix $\hat{\mathbf{R}}_{x}$.

b) Perform eigen-decomposition on $\hat{\mathbf{R}}_{x}$.

c) Use the MDL criterion (or AIC criterion) to estimate the rank of $\dot{\mathbf{R}}_{x}$ (or equivalently the rank of $\mathbf{R}_{s}$ ), say $\hat{p}^{\prime}$, then obtain $\hat{\mathbf{E}}_{s}, \hat{\sigma}^{2}$, and $\hat{\boldsymbol{\Lambda}}_{s}$.

d) Compute the estimate of the noise-free covariance matrix by

$$
\hat{\mathbf{R}}_{x}=\hat{\mathbf{E}}_{s} \mathbf{W} \hat{\mathbf{E}}_{s}^{H}
$$

where $\mathbf{W}=\hat{\Lambda}_{s}-\hat{\sigma}^{2} \mathbf{I}$.

e) Apply the forward/backward spatial smoothing algorithm to $\hat{\mathbf{R}}_{x}$, and obtain $\hat{\tilde{\mathbf{R}}}_{x}$

$$
\hat{\tilde{\mathbf{R}}}_{x}=\frac{1}{2 K} \sum_{k=1}^{K} \mathbf{Z}_{k} \hat{\dot{\mathbf{R}}}_{x} \mathbf{Z}_{k}^{T}+\mathbf{Q}_{k} \hat{\dot{\mathbf{R}}}_{x}^{*} \mathbf{Q}_{k}^{T}
$$

where $Z_{k}$ and $Q_{k}$ are given in (14), (15), and (16). Here, we assume that the source coherent structure [18] is known. Thus, we know how to choose a proper $K$.

\section{Detection of Coherent Signals}

Since coherent signals are decorrelated by WSS, the number of signals can be determined due to the special structure of the eigenvalues of $\tilde{\mathbf{R}}_{x}$, the noise-free spatially-smoothed covariance matrix. In fact, the number of signals is equal to the rank of $\tilde{\mathbf{R}}_{x}$. In practical applications, the $\tilde{\mathbf{R}}_{x}$ estimate is used, and is designated as $\hat{\tilde{\mathbf{R}}}_{x}$. If the number of snapshots is finite, the rank of $\hat{\tilde{\mathbf{R}}}_{x}$ is not equal to the number of signals. Our goal here, is to estimate the number of signals (or equivalently the rank of the matrix $\tilde{\mathbf{R}}_{x}$ ) using $\hat{\tilde{\mathbf{R}}}_{x}$. We first show that the noise eigenvalues of $\hat{\tilde{\mathbf{R}}}_{x}$ are more accurate than the noise eigenvalues of $\hat{\overline{\mathbf{R}}}_{x}$. Then, a novel modification of the MDL criterion is proposed to take advantage of this greater accuracy.

\section{A. The Accuracy of Obtained Eigenvalues}

As shown in (18), we compute eigen-decomposition of $\hat{\tilde{\mathbf{R}}}_{x}$ and obtain

$$
\hat{\tilde{\mathbf{R}}}_{x}=\sum_{k=1}^{m^{\prime}} \hat{\tilde{\lambda}}_{k} \hat{\tilde{e}}_{k} \hat{\tilde{e}}_{k}^{H}
$$

where $\hat{\tilde{\lambda}}_{1}>\hat{\tilde{\lambda}}_{2}>\cdots>\hat{\tilde{\lambda}}_{m^{\prime}} \cdot m^{\prime}$ is the number of sensors of a subarray. Then, we have the following lemma.

Lemma 1: Let $\left\{\hat{\bar{\lambda}}_{s}, s=1,2, \cdots, p\right\}$ denote the signal eigenvalues of $\hat{\overline{\mathbf{R}}}_{x}$, and $\left\{\hat{\bar{\lambda}}_{n}, n=p+1, p+2, \cdots, m^{\prime}\right\}$ denote the noise eigenvalues of $\hat{\overline{\mathbf{R}}}_{x}$. Let $\left\{\hat{\tilde{\lambda}}_{s}, s=1,2, \cdots, p\right\}$ denote the signal eigenvalues of $\hat{\tilde{\mathbf{R}}}_{x}$, and $\left\{\hat{\tilde{\lambda}}_{n}, n=p+1, p+\right.$ $\left.2, \cdots, m^{\prime}\right\}$ denote the noise eigenvalues of $\hat{\tilde{\mathbf{R}}}_{x}$. We have

$$
\begin{gathered}
\operatorname{Var}\left\{\hat{\tilde{\lambda}}_{s}\right\} \approx \operatorname{Var}\left\{\hat{\bar{\lambda}}_{s}\right\} \\
\operatorname{Var}\left\{\hat{\tilde{\lambda}}_{n}\right\} \ll \operatorname{Var}\left\{\hat{\bar{\lambda}}_{n}\right\}
\end{gathered}
$$

where $\operatorname{Var}\{y\}$ denotes the variance of $y$.
TABLE I

Variances of the Spatially Smoothed Covariance Matrix Eigenvalues

\begin{tabular}{c|c|c|c|c|c|c|c|c|c}
\hline \hline & \multicolumn{4}{|c|}{ Signal Eigenvalues } & \multicolumn{5}{c}{ Noise Eigenvalues } \\
\cline { 2 - 10 } & $\bar{\lambda}_{1}$ & $\bar{\lambda}_{2}$ & $\bar{\lambda}_{3}$ & $\bar{\lambda}_{4}$ & $\tilde{\lambda}_{5}$ & $\tilde{\lambda}_{6}$ & $\tilde{\lambda}_{7}$ & $\tilde{\lambda}_{8}$ & $\bar{\lambda}_{9}$ \\
\hline Variance & 2.979 & 0.426 & 0.277 & 0.229 & 0.027 & 0.022 & 0.016 & 0.017 & 0.018 \\
\hline
\end{tabular}

TABLE II

VARIANCES OF THE NOISE-FreE SPATIALLY Smoothed Covariance Matrix Eigenvalues

\begin{tabular}{c|c|c|c|c|c|c|c|c|c}
\hline \hline & \multicolumn{4}{|c|}{ Signal Eigenvalues } & \multicolumn{5}{c}{ Noise Eigenvalues } \\
\cline { 2 - 9 } & $\tilde{\lambda}_{1}$ & $\tilde{\lambda}_{2}$ & $\tilde{\lambda}_{3}$ & $\tilde{\lambda}_{4}$ & $\tilde{\bar{\lambda}}_{5}$ & $\tilde{\dot{\lambda}}_{6}$ & $\tilde{\lambda}_{7}$ & $\tilde{\lambda}_{8}$ & $\tilde{\lambda}_{9}$ \\
\hline Variance & 2.947 & 0.403 & 0.262 & 0.186 & 0.002 & 0.001 & 0.000 & 0.000 & 0.000 \\
\hline
\end{tabular}

Proof: Recall that $\hat{\overline{\mathbf{R}}}_{x}$ is obtained through applying the smoothing transformation to $\hat{\mathbf{R}}_{x}\left(\hat{\mathbf{R}}_{x}=\hat{\mathbf{E}}_{s} \hat{\mathbf{\Lambda}}_{s} \hat{\mathbf{E}}_{s}^{H}+\right.$ $\left.\hat{\mathbf{E}}_{n} \hat{\mathbf{\Lambda}}_{n} \hat{\mathbf{E}}_{n}^{H}\right) . \hat{\tilde{\mathbf{R}}}_{x}$ is obtained through applying the smoothing transformation to $\hat{\mathbf{R}}_{x}\left(\hat{\mathbf{R}}_{x}=\hat{\mathbf{E}}_{s}\left(\hat{\mathbf{\Lambda}}_{s}-\hat{\sigma}^{2} \mathbf{I}\right) \hat{\mathbf{E}}_{s}^{H}\right)$.

Equation (30) is necessary because perturbations of signal eigenvalues, $\hat{\tilde{\lambda}}_{s}$ and $\hat{\bar{\lambda}}_{s}$, result mainly from perturbation of $\hat{\mathbf{E}}_{s} \hat{\mathbf{\Lambda}}_{s} \hat{\mathbf{E}}_{s}^{H}$; and such perturbation exists in both $\hat{\tilde{\mathbf{R}}}_{x}$ and $\hat{\overline{\mathbf{R}}}_{x}$. Equation (31) is necessary because perturbations of noise eigenvalues, $\hat{\tilde{\lambda}}_{n}$ and $\hat{\bar{\lambda}}_{n}$, result mainly from perturbation of $\hat{\mathbf{E}}_{n} \hat{\mathbf{\Lambda}}_{n} \hat{\mathbf{E}}_{n}^{H}$; and such perturbation exists only in $\hat{\overline{\mathbf{R}}}_{x}$. Also, note that the noise eigenvalues are also slightly affected by perturbation of $\hat{\mathbf{E}}_{s} \hat{\Lambda}_{s} \hat{\mathbf{E}}_{s}^{H}$. Thus, $\operatorname{Var}\left\{\hat{\tilde{\lambda}}_{n}\right\} \neq 0$, but $\operatorname{Var}\left\{\hat{\tilde{\lambda}}_{n}\right\} \approx 0$.

We tested the lemma as follows. The uniformly spaced linear array under consideration consisted 10 equally spaced omnidirectional sensors $(m=10)$. The distance between two neighboring sensors was one half the signal wavelength. Four equal strength signals $(p=4)$, emanating from the far field, impinged on the array at distinct directions $0^{\circ}, 10^{\circ}, 20^{\circ}$, and $30^{\circ}$ w.r.t. the broadside of the array. The signals at $0^{\circ}$ and $10^{\circ}$ were coherent as were signals at $20^{\circ}$ and $30^{\circ}$. Thus, $p^{\prime}=2$. The signal-to-noise ratio, defined as the power of each signal to the noise power at each sensor, $\sigma^{2}$, was $-4 \mathrm{~dB}$. The forward/backward spatial smoothing scheme was used with each subarray containing nine sensors $\left(m^{\prime}=9, K=2\right)$. The sample covariance matrix, $\hat{\mathbf{R}}_{x}$, was formed after 100 snapshots, and $\hat{\bar{\lambda}}_{k}$ and $\hat{\tilde{\lambda}}_{k}, k=1,2, \cdots, 9$ were computed.

Based on 100 independent experiments, we computed the variances of these eigenvalues. The results are shown in Tables I and II. The values in Tables I and II reveal that the $\tilde{\hat{\lambda}}_{k}$ was more accurate than $\hat{\bar{\lambda}}_{k}$, for $k=5, \cdots, 9$. Thus, we may attempt to improve detection performance using $\hat{\bar{\lambda}}_{k}$.

\section{B. The Modified MDL Criterion}

$\hat{\tilde{\lambda}}_{k}$ cannot be put into the the $\overline{\mathrm{MDL}}$ criterion directly, because the structure of $\tilde{\lambda}_{k}$ differs from the structure of $\bar{\lambda}_{k}$ in that $\tilde{\lambda}_{k}$ is

$$
\begin{aligned}
& \qquad \tilde{\lambda}_{1}>\tilde{\lambda}_{2}>\cdots>\tilde{\lambda}_{p}>\tilde{\lambda}_{p+1}=\tilde{\lambda}_{p+2}=\cdots \tilde{\lambda}_{m^{\prime}}=0 \\
& \text { while } \bar{\lambda}_{k} \text { is } \\
& \qquad \bar{\lambda}_{1}>\bar{\lambda}_{2}>\cdots>\bar{\lambda}_{p}>\bar{\lambda}_{p+1}=\bar{\lambda}_{p+2}=\cdots \bar{\lambda}_{m^{\prime}}=\sigma^{2} .
\end{aligned}
$$


It can be shown that the structure of $\tilde{\lambda}_{k}+\sigma^{2}$ is equivalent to the structure of $\bar{\lambda}_{k}$. This indicates that we may put $\tilde{\lambda}_{k}+\hat{\sigma}^{2}$ instead of $\hat{\bar{\lambda}}_{k}$ into the $\overline{\mathrm{MDL}}$ criterion. However, the detection performance seems unimproved by this kind of substitution. Below, we propose a novel method which improves performance a great deal.

In accordance with Lemma 1, we propose a new detection method that put

$$
\check{\lambda}_{k}=M \cdot \hat{\tilde{\lambda}}_{k}+\hat{\sigma}^{2}
$$

instead of $\hat{\bar{\lambda}}_{k}$ into the $\overline{\mathrm{MDL}}$ criterion, where $M$ is a scalar greater than unity. $M$ is chosen to satisfy

$$
\operatorname{Var}\left\{\check{\lambda}_{n}\right\} \approx \operatorname{Var}\left\{\hat{\bar{\lambda}}_{n}\right\} \text {. }
$$

Naturally

$$
\begin{aligned}
E\left\{\check{\lambda}_{n}\right\} & =M \cdot E\left\{\hat{\bar{\lambda}}_{n}-\hat{\sigma}^{2}\right\}+E\left\{\hat{\sigma}^{2}\right\} \\
& =M \cdot 0+\sigma^{2} \\
& =\sigma^{2}=E\left\{\hat{\bar{\lambda}}_{n}\right\} .
\end{aligned}
$$

On the other hand, the signal eigenvalues exhibit the following properties

$$
\begin{aligned}
E\left\{\check{\lambda}_{s}\right\} & =M \cdot E\left\{\hat{\bar{\lambda}}_{s}-\hat{\sigma}^{2}\right\}+E\left\{\hat{\sigma}^{2}\right\} \\
& =(M-1) E\left\{\hat{\bar{\lambda}}_{s}-\hat{\sigma}^{2}\right\}+E\left\{\hat{\bar{\lambda}}_{s}\right\}-E\left\{\hat{\sigma}^{2}\right\}+E\left\{\hat{\sigma}^{2}\right\} \\
& =E\left\{\hat{\bar{\lambda}}_{s}\right\}+(M-1) E\left\{\hat{\bar{\lambda}}_{s}-\hat{\sigma}^{2}\right\} \\
& >E\left\{\hat{\bar{\lambda}}_{s}\right\} .
\end{aligned}
$$

From (36) and (37), we conclude that signal eigenvalues are enlarged while noise eigenvalues remain the same in the mean. As a result, the new method can improve detection performance. The question of how to choose $M$ remains. As $M$ increases, $\check{\lambda}_{s}$ is enlarged; the perturbation of $\check{\lambda}_{n}$, however, is also increased. Thus, an optimum value for $M$ exists. In simulations, we found it advisable to set $M$ to be the number of subarrays used in the spatial smoothing scheme. For the forward/backward smoothing described in Section II-B, we set $M$ to be $2 K$. We now summarize (as follows) the detection method which follows from the weighted subspace smoothing scheme presented in Section II-C:

f) Perform eigen-decomposition on $\hat{\tilde{\mathbf{R}}}_{x}$ to obtain $\hat{\tilde{\lambda}}_{k}$.

g) The number of signals is then determined as that value, $k$, which minimizes the criterion

$$
\begin{aligned}
\overline{\mathrm{MDL}}_{w}(k)= & N\left(m^{\prime}-k\right) \log \left(\frac{\tilde{a}(k)}{\tilde{g}(k)}\right) \\
& +\frac{1}{2} k\left(2 m^{\prime}-k\right) \log N
\end{aligned}
$$

where

$$
\begin{aligned}
& \tilde{a}(k) \triangleq \frac{1}{m^{\prime}-k} \sum_{i=k+1}^{m^{\prime}}\left(2 K \hat{\tilde{\lambda}}_{i}+\hat{\sigma}^{2}\right) \\
& \tilde{g}(k) \triangleq\left(\prod_{i=k+1}^{m^{\prime}}\left(2 K \hat{\tilde{\lambda}}_{i}+\hat{\sigma}^{2}\right)\right)^{1 /\left(m^{\prime}-k\right)} .
\end{aligned}
$$

Here, $k \in\left\{0,1, \cdots, m^{\prime}-1\right\}$.

\section{Simulations}

Computer simulations were carried out to compare detection performances among the $\overline{\mathrm{MDL}}_{w}$ criterion, the $\overline{\mathrm{MDL}}$ criterion, and the WSF detection scheme. Examples 1.1, 1.2, and 1.3 (below) show that detection performance of the $\overline{\mathrm{MDL}}_{w}$ criterion is much higher than the $\overline{\mathrm{MDL}}$ criterion and the WSF detection scheme, especially when more signals are coherent. Example 2 shows that a best value of $M$ for the $\overline{\mathrm{MDL}}_{w}$ criterion exists. If $M$ is set to be a too small value, detection performance is hardly improved. If $M$ is too large, the $\overline{\mathrm{MDL}}_{w}$ criterion tends to overestimate the number of signals. In Examples 3.1 and 3.2, we explore how unknown spatially-correlated noise affects these methods. Although the performances of all these methods deteriorate due to the spatially-correlated noise, the $\overline{\mathrm{MDL}}_{w}$ criterion turns to be the most robust one.

Remark: For the WSF detection scheme, we use the MODE-2 method proposed in [27] instead of using the modified Gauss-Newton algorithm, which was called the modified variable projection (MVP) algorithm in [29], to minimize the WSF cost function. One advantage of using MODE-2 is that we do not need to use ESPRIT or the alternating projection method to initiate DOA estimates for the MVP algorithm. Another advantage is that MODE-2 doesn't require a subjective steplength. Moreover, in all our simulations using the MODE-2 method to minimize the WSF cost function performs much better than using the MVP algorithm to do the same minimization. Note also, that the threshold for the hypothesis test within the WSF detection scheme is selected as the value at level 0.995 from an appropriate chi-square distribution (see [29] for more details).

Example 1.1: In this example, a 10-sensor uniformlyspaced linear array with one half-wavelength inter-sensor spacing was employed. Five narrow-band signals with equal power impinged on the array, from the far field, at distinct directions $-20^{\circ},-10^{\circ}, 0^{\circ}, 10^{\circ}$, and $20^{\circ}$ w.r.t. the broadside of the array. Signals at $0^{\circ}, 10^{\circ}$, and $20^{\circ}$ were coherent. Signals at $-20^{\circ}$ and $-10^{\circ}$ were uncorrelated with all other signals. Spatial-white Gaussian was an additive. The signalto-noise ratio (SNR) was defined as the ratio of each signal power to the noise power at each sensor. We then used 100 snapshots to construct the sample covariance matrix $\hat{\mathbf{R}}_{x}$. Since the rank of the exact signal covariance $\mathbf{R}_{s}$ was $3(<5)$, the MDL criterion failed to detect five signals. We employed the original spatial smoothing scheme or the proposed WSS to decorrelate the signal coherency, and then both $\overline{\mathrm{MDL}}$ and $\overline{\mathrm{MDL}}_{w}$ criteria did detect five signals, provided that SNR was sufficiently high. In this example, the forward/backward smoothing transformation was used with smoothing degree $K=3$. Thus, each subarray consisted of eight sensors, and $M=6$ for $\overline{\mathrm{MDL}}_{w}$ criterion. We also used the WSF detection scheme to detect these five signals. To examine which criterion possessed better performance, we varied SNR and calculated detection probabilities for each method. One hundred independent Monte Carlo trials were used to calculate the detection probability for each SNR. The results are presented in Fig. 1. We plot the detection probabilities as a function of SNR and observe that the $\overline{\mathrm{MDL}}_{w}$ criterion 


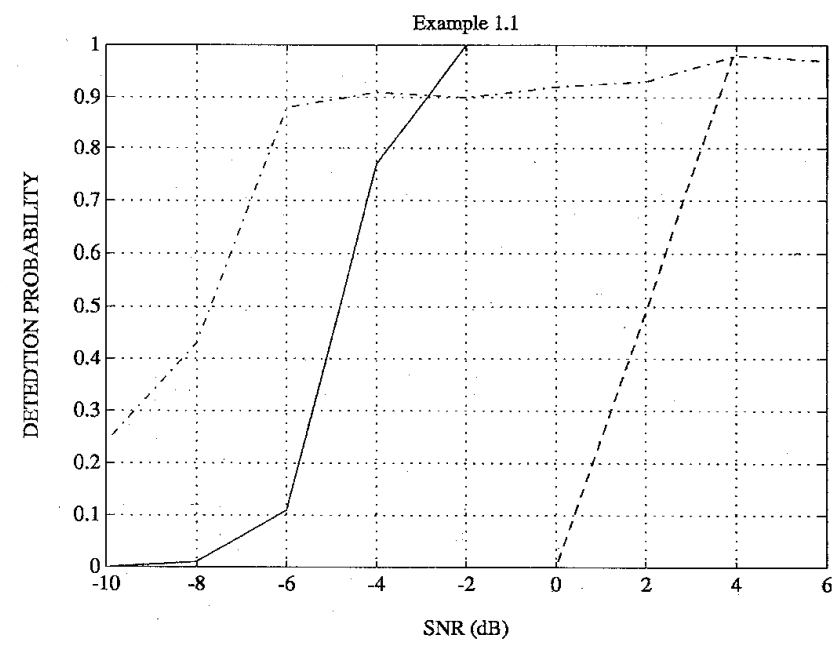

Fig. 1. The detection probabilities versus SNR. Results of Example 1.1-three coherent signals. Solid line: The $\overline{\mathrm{MDL}}_{w}$ criterion. Dashed line: The $\overline{M D L}$ criterion. Dot dash line: The WSF scheme.

TABLE III

The Number of CoRrect Detections (OUT OF 100) For VARIOUS SUBARRAY SIZES

\begin{tabular}{|c|c|c|c|c|}
\hline & \multicolumn{3}{|c|}{ subarray size $m^{\prime}$} \\
\hline & & $m^{\prime}=9$ & $m^{\prime}=8$ & $m^{\prime}=7$ \\
\hline \multirow[t]{2}{*}{$\mathrm{SNR}=-2$} & $\overline{\mathrm{MDL}}_{w}$ & 13 & 100 & 8 \\
\hline & $\overline{\mathrm{MDL}}$ & 0 & 0 & 0 \\
\hline \multirow{2}{*}{$\mathrm{SNR}=0$} & $\overline{\mathrm{MDL}}_{w}$ & 9 & 100 & 75 \\
\hline & $\overline{\mathrm{MDL}}$ & 0 & 0 & 0 \\
\hline \multirow[t]{2}{*}{$\mathrm{SNR}=2$} & $\overline{\mathrm{MDL}}_{w}$ & 100 & 100 & 100 \\
\hline & $\overline{\mathrm{MDL}}$ & 0 & 49 & 0 \\
\hline \multirow[t]{2}{*}{$\mathrm{SNR}=4$} & $\overline{\mathrm{MDL}}_{w}$ & 100 & 100 & 100 \\
\hline & $\overline{\mathrm{MDL}}$ & 17 & 100 & 0 \\
\hline
\end{tabular}

outperformed the $\overline{\mathrm{MDL}}$ criterion by about $6.5 \mathrm{~dB}$. When SNR $>-3 \mathrm{~dB}$, the $\overline{\mathrm{MDL}}_{w}$ criterion outperformed the WSF detection scheme. When SNR $<-3 \mathrm{~dB}$, although the WSF detection scheme was better than the $\overline{\mathrm{MDL}}_{w}$ criterion, it only exhibited detection probability below $90 \%$. Obviously, when SNR $<-3 \mathrm{~dB}$, we prefer the WSF detection scheme. Thus, how to combine these two methods to maximize the detection probability for all SNR's is an interesting issue, but is beyond the scope of this paper.

We are also interested in the effect of subarray size. In Table III, the number of correct detections (out of 100) is listed in a column corresponding to different subarray sizes for various SNR's. We find that for either $\overline{\mathrm{MDL}}_{w}$ or $\overline{\mathrm{MDL}}$ criterion, the best choice of subarray size was eight. Moreover, $\overline{\mathrm{MDL}}_{w}$ always outperformed $\overline{\mathrm{MDL}}$ criterion, regardless of subarray size.

Example 1.2: This example further checked performance improvement of $\overline{\mathrm{MDL}}_{w}$ criterion. Simulation conditions were

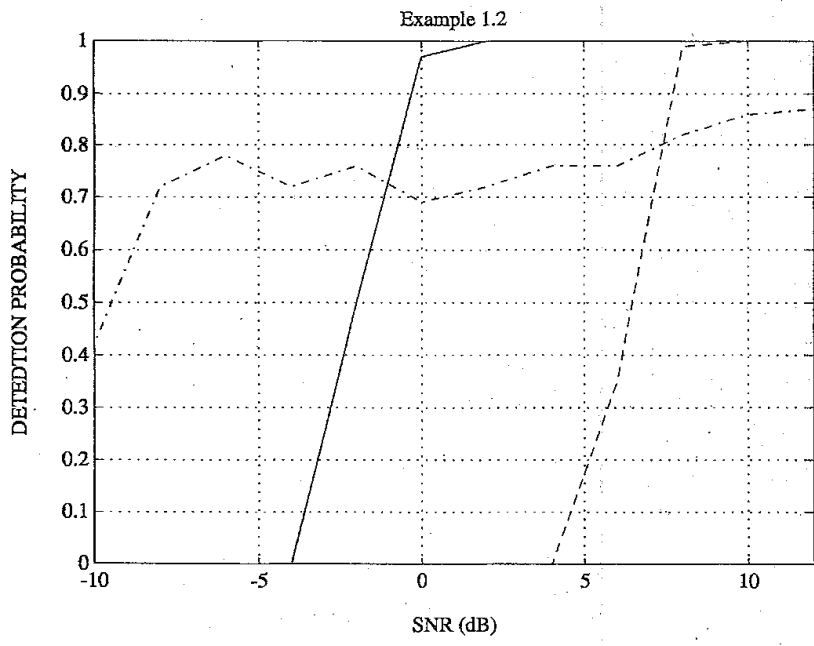

Fig. 2. The detection probabilities versus SNR. Results of Example 1.2--four coherent signals. Solid line: The $\overline{\mathrm{MDL}}_{w}$ criterion. Dashed line: The $\overline{\mathrm{MDL}}$ criterion. Dot dash line: The WSF scheme.

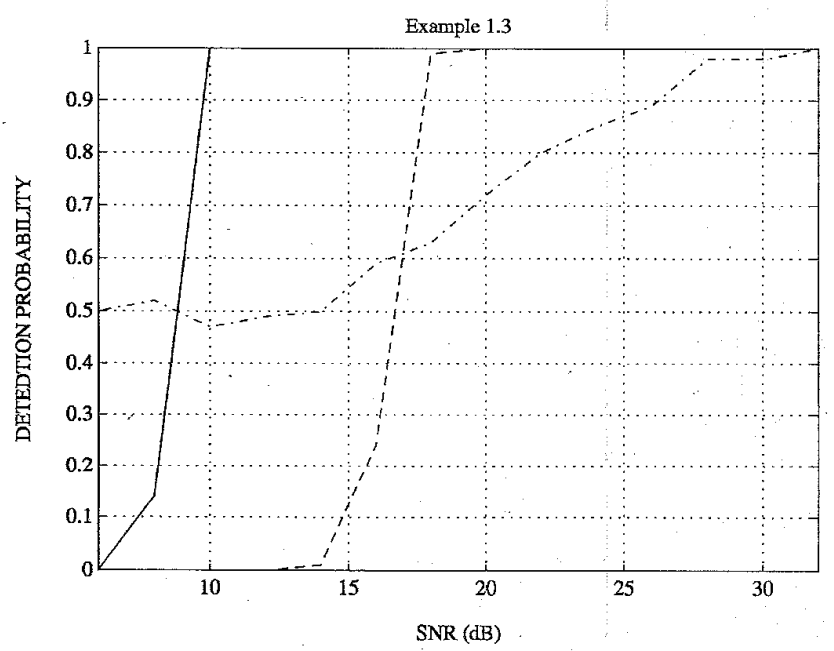

Fig. 3. The detection probabilities versus SNR. Results of Example 1.3-five coherent signals. Solid line: The $\overline{\mathrm{MDL}}_{w}$ criterion. Dashed line: The $\overline{\mathrm{MDL}}$ criterion. Dot dash line: The WSF scheme.

the same as those in Example 1.1, except the signal coherency-signals at $-10^{\circ}, 0^{\circ}, 10^{\circ}$, and $20^{\circ}$ were coherent and the signal at $-20^{\circ}$ was uncorrelated with all other signals. In this example, the best choice of subarray size was seven. Simulation results are shown in Fig. 2. The $\overline{\mathrm{MDL}}_{w}$ criterion outperformed the $\overline{\mathrm{MDL}}$ criterion by about $8.5 \mathrm{~dB}$. When SNR $>-1 \mathrm{~dB}$, the $\overline{\mathrm{MDL}}_{w}$ criterion outperformed the WSF detection scheme. When SNR $>8 \mathrm{~dB}$, even the $\overline{\mathrm{MDL}}$ criterion outperformed the WSF detection scheme. When SNR $<-1$ $\mathrm{dB}$, although the WSF detection scheme was better than the $\overline{\mathrm{MDL}}_{w}$ criterion, it only exhibited detection probability below $80 \%$.

Example 1.3: Simulation conditions were the same as those in Example 1.2, except that all signals were coherent. Simulation results are shown in Fig. 3. The $\overline{\mathrm{MDL}}_{w}$ criterion outperformed the $\overline{\mathrm{MDL}}$ criterion by about $8 \mathrm{~dB}$. The WSF 
TABLE IV

The Number of Correct Detections (Out OF 100) FOR DIFFERENT VALUES OF $M$

\begin{tabular}{l|c|ccccccccccc}
\hline & \multirow{2}{*}{$\overline{\mathrm{MDL}}$} & \multicolumn{10}{|c}{$\overline{\mathrm{MDL}}_{u^{\prime}}$ with different $M$} \\
\cline { 3 - 15 } & & $M=1$ & 2 & 3 & 4 & 5 & 6 & 7 & 8 & 9 & 10 & 11 \\
\hline $\mathrm{SNR}=-8$ & 0 & 0 & 0 & 0 & 2 & 4 & 4 & 6 & 9 & 10 & 11 & 15 \\
$\mathrm{SNR}=-6$ & 0 & 0 & 0 & 9 & 36 & 55 & 60 & 60 & 63 & 61 & 61 & 56 \\
$\mathrm{SNR}=-4$ & 0 & 0 & 32 & 84 & 97 & 98 & 98 & 99 & 96 & 95 & 93 & 90 \\
$\mathrm{SNR}=-2$ & 9 & 3 & 99 & 100 & 100 & 100 & 100 & 98 & 97 & 92 & 91 & 90 \\
$\mathrm{SNR}=-0$ & 93 & 93 & 100 & 100 & 100 & 100 & 99 & 97 & 97 & 97 & 95 & 95 \\
$\mathrm{SNR}=2$ & 100 & 100 & 100 & 100 & 100 & 100 & 99 & 99 & 99 & 99 & 96 & 94 \\
$\mathrm{SNR}=4$ & 100 & 100 & 100 & 100 & 100 & 100 & 100 & 99 & 99 & 99 & 96 & 93 \\
$\mathrm{SNR}=6$ & 100 & 100 & 100 & 100 & 100 & 100 & 100 & 100 & 100 & 100 & 95 & 93 \\
$\mathrm{SNR}=8$ & 100 & 100 & 100 & 100 & 100 & 100 & 100 & 99 & 97 & 97 & 95 & 95 \\
$\mathrm{SNR}=10$ & 100 & 100 & 100 & 100 & 100 & 100 & 99 & 99 & 99 & 98 & 96 & 96 \\
$\mathrm{SNR}=12$ & 100 & 100 & 100 & 100 & 100 & 100 & 100 & 100 & 100 & 99 & 98 & 95 \\
\hline
\end{tabular}

detection scheme turned out to be an unsatisfactory method, which exhibited poor detection probability. In this case, we even prefer the $\overline{M D L}$ criterion to the WSF detection scheme.

Judging from Examples 1.1, 1.2, and 1.3, we conclude that the $\overline{\mathrm{MDL}}_{w}$ criterion is the best criterion for detecting coherent signals impinging on a uniformly-spaced linear array.

Example 2: In this example, we examined the effect of the scalar $M$, which enlarged the distance between signal and noise eigenvalues. The same simulation conditions as those in Example 1.1 were used, except the signal coherency: signals at $-10^{\circ}$ and $0^{\circ}$ were coherent, signals at $10^{\circ}$ and $20^{\circ}$ were coherent. Each subarray consisted nine sensors. In Table IV, the number of correct detections (out of 100) is listed in a column corresponding to the $\overline{\mathrm{MDL}}$ criterion and the $\overline{\mathrm{MDL}}_{w}$ criterion with different value of $M$ for various SNR's. We observe that when $M$ was set to be $1, \overline{\mathrm{MDL}}_{w}$ criterion did not perform better than $\overline{\mathrm{MDL}}$ criterion. When SNR $=-2$, the performance of the $\overline{\mathrm{MDL}}_{w}$ criterion was even inferior to the $\overline{\mathrm{MDL}}$ criterion. Suitable value of $M$ was between $3-5$. Note that in the example, the number of subarrays used in smoothing transformation was four. This validates that an advisable choice of $M$ is the number of subarrays used in smoothing transformation. When $M \geq 6$, the performance of the $\overline{\mathrm{MDL}}_{w}$ criterion deteriorated. In fact, when $M=7,8,9,10$, or 11 , the $\overline{\mathrm{MDL}}_{w}$ criterion tended to overestimate the number of signals.

Example 3.1: We used this and the next example to see the effect of spatially-correlated noise on all the ad hoc detection methods. All simulation conditions of this example were the same as Example 1.1, except that the additive noise was spatially correlated and the SNR was fixed at 8 $\mathrm{dB}$. The spatially-correlated noise had exponentially decaying correlation among sensors. The noise covariance matrix had $i k$ th element

$$
Q_{i k}=\sigma^{2} \alpha^{\|i-k\|} e^{j(\pi / 2)(i-k)}
$$

where $\sigma^{2}$ was adjusted to give the desired SNR and $\alpha$ became an indicator which indicated how severe the spatial correlation was. When $\alpha=0$, there was no correlation among sensors. As $\alpha$ grew up, the correlation increased. Note that this noise correlation model was the same as used in [31]-[33]. No spatial prewhiten of the data was performed, since the noise covariance was unknown. Fig. 4 shows the performance degradation due to increasing $\alpha$. When $\alpha=0$,

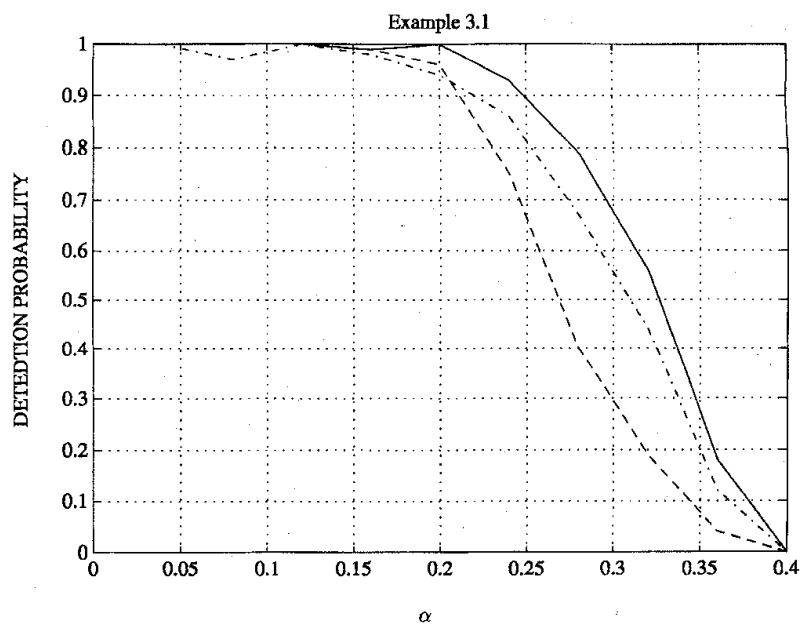

Fig. 4. The detection probabilities in spatially correlated noise fields. Results of Example 3.1-three coherent signals. Solid line: The $\overline{\mathrm{MDL}}_{w}$ criterion. Dashed line: The $\overline{M D L}$ criterion. Dot dash line: The WSF scheme.

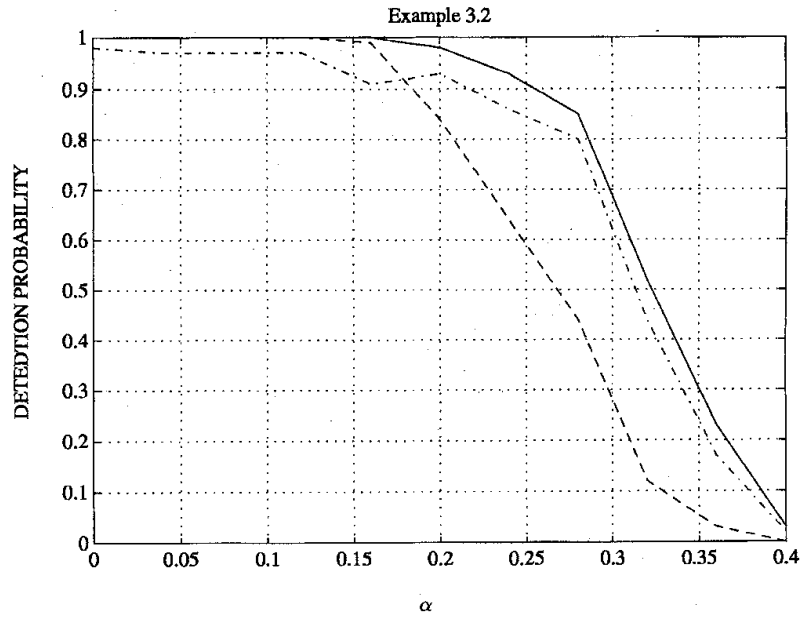

Fig. 5. The detection probabilities in spatially correlated noise fields. Results of Example 3.2-four coherent signals. Solid line: The $\overline{\mathrm{MDL}}_{w}$ criterion. Dashed line: The $\overline{\mathrm{MDL}}$ criterion. Dot dash line: The WSF scheme.

all these methods exhibited $100 \%$ detection probability. When $\alpha=0.25$, the detection probability of the $\overline{\mathrm{MDL}}_{w}$ criterion was about $90 \%$, the WSF detection scheme about $80 \%$, and the $\overline{\mathrm{MDL}}$ criterion about $65 \%$. Although the detection capabilities of all these methods degraded due to spatial correlation among sensors, the $\overline{\mathrm{MDL}}_{w}$ criterion turned out to be the most robust method.

Example 3.2: We checked, further, the robustness using this example. All simulation conditions of this example were the same as Example 1.2, except that the additive noise was spatially correlated and the SNR was fixed at $16 \mathrm{~dB}$. The noise correlation model used in Example 3.1 was used in this example, too. Fig. 5 shows the performance degradation due to increasing $\alpha$. Again, the $\overline{\mathrm{MDL}}_{w}$ criterion was the most robust method, then the WSF detection scheme, finally the $\overline{\mathrm{MDL}}$ criterion. Actually, we observe that the performance degradation of the $\overline{\mathrm{MDL}}_{w}$ criterion and the WSF detection 
scheme was similar and more mild than the $\overline{\mathrm{MDL}}$ criterion. This occured probably because both these two methods took the advantage of the weighted signal subspaces while the $\overline{\mathrm{MDL}}$ criterion did not.

\section{CONCLUSION}

We have proposed a new approach to determining the number of coherent signals impinging on uniformly spaced linear arrays. It combines a new version of spatial smoothing and a new version of the MDL criterion, and greatly improves detection performance when it compares with the method that directly combines the MDL criterion and the spatial smoothing. When there are many coherent signals, the proposed approach also performs much better than the WSF detection scheme, which is much more complicated than the proposed approach. We call the new spatial smoothing approach weighted subspace smoothing. Analysis and simulations show the noise eigenvalues obtained with the weighted subspace smoothing are more accurate than those obtained with the original spatial smoothing scheme. As a result, detection performance can be improved by using these more accurate eigenvalues. One of the contributions of this paper is the establishment of a new version of the MDL criterion which accounts for the greater accuracy and leads to great performance improvement. An important parameter $M$ of the proposed $\overline{\mathrm{MDL}}_{w}$ criterion enlarges the distance between signal and noise eigenvalues. This is the reason why detection performance could be improved. However, the question of how to choose the optimum value of $M$ remains. We hope to report a systemic solution for this in future work. Weighted subspace smoothing can also be used to improve DOA (direction-ofarrival) estimation. In [30], the weighting matrix $W$ was set to be $\hat{\mathbf{\Lambda}}_{s}$, and more correct DOA estimates were obtained. Taking view of the results reported in this paper and in [30], we find that it is advisable to use weighted subspace smoothing for both signal-detection and DOA-estimation of coherent signals impinging on uniformly-spaced linear arrays.

\section{ACKNOWLEDGMENT}

The authors would like to thank T.-S. Lee, Y.-L. Su, and the anonymous reviewers for their valuable comments and suggestions.

\section{REFERENCES}

[1] R. O. Schmidt, "Multiple emitter location and signal parameter estimation," in Proc., RADC Spectrum Estimat. Workshop, Griffiths AFB, NY, 1979, pp. 243-258; also in IEEE Antennas Propagat., vol. AP-34, no. 3, pp. 276-380, Mar. 1986.

[2] D. W. Tufts and R. Kumaresan, "Estimating the angle of arrival of multiple plane waves," IEEE Trans. Aerosp. Electron. Syst., vol. AES-19, pp. 135-139, Jan. 1983.

[3] R. Roy and T. Kailath, "ESPRIT-Estimation of signal parameter via rotational invariance," IEEE Trans. Acoust., Speech, Signal Processing, vol. 37, pp. 984-995, July 1989.

[4] I. Ziskind and M. Wax, "Maximum likelihood localization of multiple sources by alternating projection," IEEE Trans. Acoust., Speech, Signal Processing, vol. 36, pp. 1553-1560, Oct. 1988.

[5] M. S. Bartlett, "A note on the multiplying factors for various $\chi^{2}$ approximations," J. Roy. Stat. Soc., ser. B, vol. 16, pp. 296-298. 1954.
[6] G. Bienvenu and L. Kopp, "Optimality of high resolution array processing using the eigensystem approach," IEEE Trans. Acoust, Speech, Signal Processing, vol. ASSP-31, pp. 1235-1247, Oct. 1983.

[7] H. Akaike, "A new look at the statistical model identification," IEEE Trans. Automat. Contr., vol. AC-19, pp. 716-723, 1974.

[8] J. Rissanen, "Modeling by shortest data description," Automatica, vol. 14, pp. $465-471,1978$

[9] M. Wax and T. Kailath, "Detection of the number of signals by information theoretic criterion," IEEE Trans. Acoust., Speech, Signal Processing, vol. 33, pp. 387-392, 1984.

[10] L. C. Zhao, P. R. Krishnaiah, and Z. D. Bai, "On detection of the number of signals in presence of white noise," J. Multivariable Anal., vol. 20, pp. $1-20,1986$.

[11] _Remarks on certain criteria for detection of number of signals," IEEE Trans. Acoust., Speech, Signal Processing, vol. ASSP-35, pp. 129-132, Feb. 1987.

[12] Y. Yin and P. Krishnaiah, "On some nonparametric methods for detection of the number of signals," IEEE Trans. Acoust., Speech, Signal Processing, vol. ASSP-35, pp. 1533-1538, 1985.

[13] H. Wang and M. Kaveh, "On the performance of signal-subspace processing-Part I: Narrow-band systems;" IEEE Trans. Acoust., Speech, Signal Processing, vol. ASSP-34, no. 5, pp. 1201-1209, Oct. 1986.

[14] M. Kaveh, H. Wang, and H. Hung, "On the theoretical performance of a class of estimators of the number of narrow-band sources," IEEE Trans. Acoust., Speech, Signal Processing, vol. ASSP-35, no. 9, pp. 1350-1352, Sept. 1987.

[15] Q. T. Zhang, K. M. Wong, P. C. Yip, and J. P. Reilly, "Statistical analysis of the performance of information theoretic criteria in the detection of the number of signals in array processing," IEEE Trans. Acoust., Speech, Signal Processing, vol. 37, no. 10, pp. 1557-1567, Oct. 1989.

[16] K. M. Wong, Q. T. Zhang, J. P. Reilly, and P. C. Yip, "On information theoretic criteria for determining the number of signals in high resolution array processing," IEEE Trans. Acoust., Speech, Signal Processing, vol. 38, no. 11, pp. 1959-1071, Nov. 1990.

[17] W. Chen, K. M. Wong, and J. P. Reilly, "Detection of the number of signal: A predicted eigen-threshold approach," IEEE Trans. Acoust., Speech, Signal Processing, vol. 39, no. 5, pp. 1088-1098, May 1991.

[18] T. J. Shan, A. Paulraj, and T. Kailath, "On smoothed rank profile tests in eigenstructure methods for directions-of-arrival estimation," IEEE Trans. Acoust., Speech, Signal Processing, vol. ASSP-35, no. 10, pp. 1377-1385, Oct. 1987.

[19] M. Wax and I. Ziskind, "Detection of the number of coherent signals by the MDL principle," IEEE Trans. Acoust., Speech, Signal Processing, vol. 37, no. 8, pp. 1190-1196, Aug. 1989.

[20] M. Wax, "Detection and localization of multiple sources via the stochastic signals model," IEEE Trans. Signal Processing, vol. 39, no. 11, pp. 2450-2456, Nov. 1991.

[21] Q. Wu and D. R. Fuhrmann, "A parametric method for determining the number of signals in narrow-band direction finding," IEEE Trans. Signal Processing, vol. 39, no. 8, pp. 1848 -1857, Aug. 1991.

[22] C. M. Cho and P. M. Djurić, "Detection and localization of multiple sources via Bayesian predictive densities," in Proc. ICASSP '93, 1993, vol. 4 , pp. $57-60$.

[23] J. E. Evans, J. R. Jognsom, and D. F. Sun, "Application of advanced signal processing techniques to angle of arrival estimate in ATC navigation and surveillance system," Rep. 582, MIT Lincoln·Lab., Lexington, MA., 1982.

[24] T. J. Shan, M. Wax, and T. Kailath, "On spatial smoothing of estimation of coherent signals," IEEE Trans. Acoust., Speech, Signal Processing, vol. ASSP-33, pp. 806-811, Aug. 1985.

[25] S. U. Pillai and B. H. Kwon, "Forward/backward spatial smoothing techniques for coherent signal identification," IEEE Trans. Acoust., Speech, Signal Processing, vol. 37, no. 1, pp. 8-15, Jan. 1989.

[26] Y. Bresler and A. Macovski, "Exact maximum likelihood parameter estimation of superimposed exponential signals in noise," IEEE Trans. Acoust., Speech, Signal Processing, vol. ASSP-34, no. 5, pp. 1081-1089, Oct. 1986.

[27] P. Stoica and K. C. Sharman, "Maximum likelihood methods for direction-of-arrival estimation," IEEE Trans. Acoust., Speech, Signal Processing, vol. 38, no. 7, pp. 1132-1143, July 1990.

[28] M. Viberg and B. Ottersten, "Sensor array processing based on subspace fitting," IEEE Trans. Signal Processing, vol. 39, no. 5, pp. 1110-1121, May 1991.

[29] M. Viberg, B. Ottersten, and T. Kailath, "Detection and estimation in sensor arrays using weighted subspace fitting,": IEEE Trans. Signal Processing, vol. 39, no. 11, pp. 2436-2448, Nov. 1991.

[30] H. Krim and J. G. Proakis, "Smoothed eigenspaced-based parameter estimation," Automatica, vol. 30, no. 1, pp. 27-38, 1994. 
[31] J. P. Reilly, K. M. Wong, and P. M. Reilly, "Direction of arrival estimation in the presence of noise with unknown, arbitrary covariance matrices," in Proc. ICASSP '89, Glasgow, Scotland, 1989, pp 2609-2612.

[32] M. Wax, "Detection and localization of multiple sources in noise with unknown covariance," IEEE Trans. Signal Processing, vol. 40, no. 1, pp. 245-249, Jan. 1992

[33] P. Stoica, M. Viberg, and B. Ottersten, "Instrumental variable approach to array processing in spatially correlated noise fields," IEEE Trans. Signal Processing, vol. 42, no. 1, pp. 121-133, Jan. 1994.

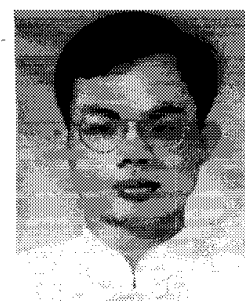

Ching-Wen Ma (S'94) was born in Chia-Yi, Taiwan, R.O.C., on May 24, 1968. He received the B.S. degree in electrical engineering from the National Taiwan Ocean University, in 1990. In 1992, he received the M.S. degree in control engineering from the National Chiao-Tung University, Taiwan, where he is currently working toward the Ph.D. degree.

His main research interests are array signal processing, adaptive signal processing, and neural networks.

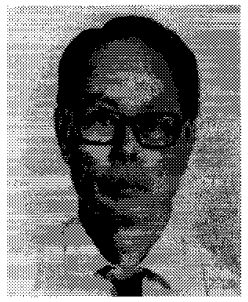

Ching-Cheng Teng was born in Taiwan in 1938. He received the B.S. degree in electrical engineering from Nation Cheng-Kung University, Taiwan, in 1961.

$\mathrm{He}$ is currently a Professor and Chairman of the department of control engineering at National Chiao-Tung University. His research interests include $H^{\infty}$ optimal control, signal processing, and fuzzy neural systems. 